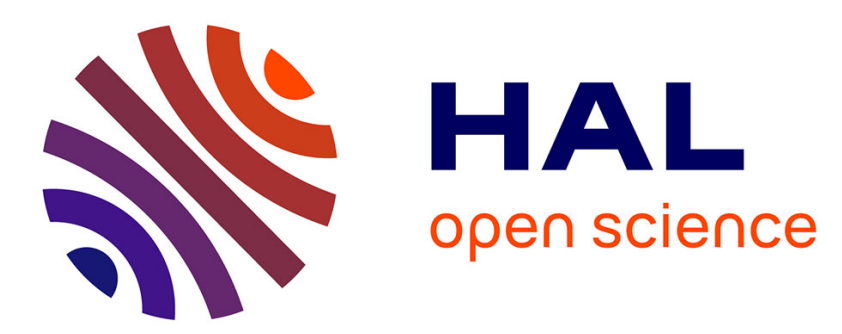

\title{
Nonlinear structural damage detection based on cascade of Hammerstein models
}

\author{
Marc Rebillat, Rafik Hajrya, Nazih Mechbal
}

\section{To cite this version:}

Marc Rebillat, Rafik Hajrya, Nazih Mechbal. Nonlinear structural damage detection based on cascade of Hammerstein models. Mechanical Systems and Signal Processing, 2014, 48 (1-2), pp. 247 - 259. 10.1016/j.ymssp.2014.03.009 . hal-01001853

\section{HAL Id: hal-01001853 \\ https://hal.science/hal-01001853}

Submitted on 5 Jun 2014

HAL is a multi-disciplinary open access archive for the deposit and dissemination of scientific research documents, whether they are published or not. The documents may come from teaching and research institutions in France or abroad, or from public or private research centers.
L'archive ouverte pluridisciplinaire HAL, est destinée au dépôt et à la diffusion de documents scientifiques de niveau recherche, publiés ou non, émanant des établissements d'enseignement et de recherche français ou étrangers, des laboratoires publics ou privés. 


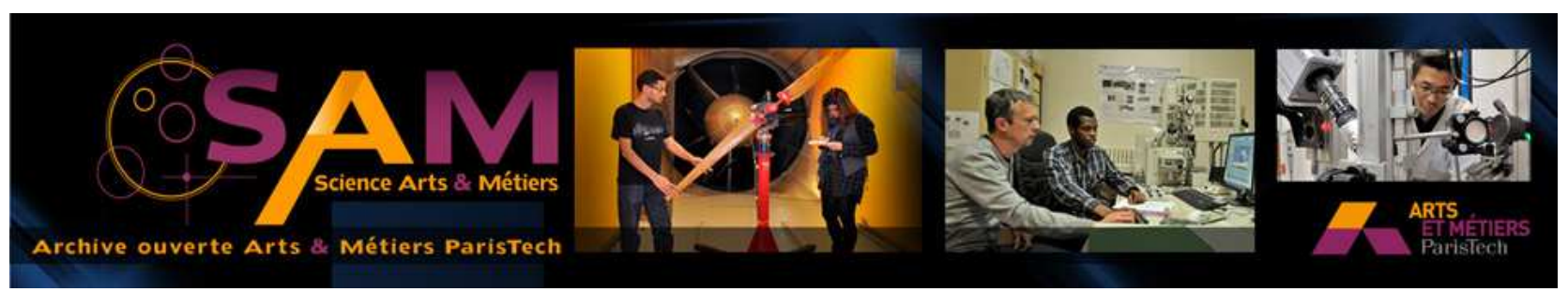

\section{Science Arts \& Métiers (SAM)}

is an open access repository that collects the work of Arts et Métiers ParisTech researchers and makes it freely available over the web where possible.

This is an author-deposited version published in: http://sam.ensam.eu

Handle ID: .http://hdl.handle.net/10985/8235

\section{To cite this version :}

Marc REBILLAT, Rafik HAJRYA, Nazih MECHBAL - Nonlinear structural damage detection based on cascade of Hammerstein models - Mechanical Systems and Signal Processing - Vol. 48, $n^{\circ} 1--$ 2, p.247 -- $259-2014$ 


\title{
Nonlinear structural damage detection based on cascade of Hammerstein models
}

\author{
Marc Rébillat \\ PIMM, Arts et Métiers ParisTech, 75013 Paris, France \\ Rafik Hajrya \\ PIMM, Arts et Métiers ParisTech, 75013 Paris, France \\ Nazih Mechbal \\ PIMM, Arts et Métiers ParisTech, 75013 Paris, France
}

\begin{abstract}
Structural damages can result in nonlinear dynamical signatures that can significantly enhance their detection. An original nonlinear damage detection approach is proposed that is based on a cascade of Hammerstein models modelisation of the structure. This model is estimated by means of the Exponential Sine Sweep Method from only one measurement. On the basis of this estimated model, the linear and nonlinear parts of the output are estimated, and two damage indexes (DIs) are proposed. The first DI is built as the ratio of the energy contained in the nonlinear part of an output versus the energy contained in its linear part. The second DI is the angle between the subspaces described by the nonlinear parts of two set of outputs after a principal component analysis. The sensitivity of the proposed DIs to the pres-
\end{abstract}

Email addresses: marc.rebillat@ensam.eu (Marc Rébillat), raf.hajrya@gmail.com (Rafik Hajrya), nazih.mechbal@ensam.eu (Nazih Mechbal) 
ence of damages as well as their robustness to noise are assessed numerically on spring-mass-damper structures and experimentally on actual composite plates with surface-mounted PZT-elements. Results demonstrate the effectiveness of the proposed method to detect nonlinear damage in nonlinear structures and in the presence of noise.

Keywords:

Structural health monitoring, non-linear system identification, damage detection.

\section{Introduction}

2

The process of implementing a damage detection strategy for aerospace, civil, and mechanical engineering is referred to as structural health monitoring (SHM). In many cases, damages that appear on complex structures (such as cracks, impacts, or delaminations) can result in nonlinear dynamical responses that may be used for damage detection [1-4]. Furthermore, complex structures often exhibit a nonlinear behavior even in their healthy states. A robust and reliable SHM system must then be able to deal with nonlinear damages, and to distinguish between their effects and inherent nonlinearities in healthy structures. Several limitations of existing methods that are facing these issues have been recently identified in a report by Farrar et al. [1]. The first problem to be addressed is that "nonlinear behavior does not generalize". This implies that the nonlinear models already in use are never general enough to encompass all the structure encountered in real life. The second problem is that "nonlinear approaches are computationally cumbersome, expensive, and requires too many parameters to be defined". Currently 
developed nonlinear models are thus not adequate for practical use of SHM systems. The work presented here attempts to face these two problems on the basis of a simple, but rather general, nonlinear model identified by means of a simple signal processing procedure.

In order to build a damage index (DI) that is sensitive to nonlinearities different approaches have already been proposed [1, 2]. Some DIs are based on a physical modeling of the damaged structure whereas some are computed without any physical assumption (black box models). Among these black-box approaches, some assume a parametric underlying signal processing model, whereas some are fully non-parametric. To feed these models, random inputs as well as deterministic broadband or narrowband inputs are used. In this paper, the focus is put on nonlinear damage detection approaches based on DIs built using a non-parametric black box model estimated using a deterministic broadband signal. There have been relatively few works in that direction. In a linear framework, some authors $[5,6]$ have shown that a nonlinear damage will impact the transmissibility functions (i.e. the frequency domain ratio between two different outputs of the system) and they used such information to detect and locate the damage. Extending the notion of transmissibility functions to nonlinear systems that can be described by Volterra series, Lang et. al $[7,8]$ were able to quantify the decrease of linearity generated by a nonlinear damage and thus to effectively detect and locate it. However, as such approaches are focusing on the loss of linearity, they do not seem to be able to deal with systems that are nonlinear in their healthy states, a fact that is quite common in real life. To overcome this drawback, several authors attempted to fit a nonlinear model to the nonlinear structure under study 
and to compare the actual and predicted outputs, or directly the model coefficients, under different damage conditions [9-12]. By doing so, they were able to detect numerically and experimentally a nonlinear damage even in an initially nonlinear structure. However, the models they used where parametric (mainly frequency domain ARX models) and thus were not easy to manipulate and neither able to model, without any a priori on it, a general nonlinear structure.

We propose here an original approach devoted to nonlinear damage detection in possibly nonlinear structures based on a simple, but rather general, nonlinear model estimated by means of standard signal processing tools. This approach is based on the assumption that the structure under study can be modeled as a cascade of Hammerstein models [13], made of $N$ branches in parallel composed of an elevation to the $n^{\text {th }}$ power followed by a linear filter called the $n^{\text {th }}$ order kernel, see Fig. 1(a). The Exponential Sine Sweep Method [14, 15], previously developed and validated by the authors for different purposes, is then used to estimate the different kernels of the model. Exponential sine sweeps are a class of sine sweeps that allow estimating a system's $N$ first kernels in a wide frequency band from only one measurement. Two damage indexes are then build on the basis of this estimated model. The first one reflects the ratio of the energy contained in the nonlinear part of the output versus the energy contained in its linear part and is specially suited for single-input single-output (SISO) systems. The second one is the angle between the subspaces described by the nonlinear parts of two set of outputs after a principal component analysis. This one is specially suited for single-input multi-output (SIMO) systems. As a first step toward the use 
of this method for SHM, the sensitivity of the proposed DIs to the presence of damages as well as their robustness to noise are assessed numerically on SISO and SIMO systems and experimentally on two actual composite plates with surface-mounted PZT-elements (one healthy and one damaged).

The cascade of Hammerstein models as well as the mathematics behind it are first described in Sec. 2. The two proposed DIs are then defined in Sec. 3. Their sensitivity to the presence of damages as well as their robustness to noise are assessed numerically in Sec 4 and experimentally in Sec. 5. A general conclusion is finally drawn in Sec. 6 .

\section{Cascade of Hammerstein models estimation using the exponen- tial sine sweep method \\ 2.1. Cascade of Hammerstein models}

A possible approach to non-linear system identification is to assume that systems have a given block-structure. Following the "sandwich" approach [13], a non-linear system can be represented as $N$ parallel branches composed of three elements in series: a static non-linear part sandwiched between two linear parts. Such systems are a subclass of Volterra systems and it can be shown that any continuous non-linear system can be approximated by such a model [16].

Cascade of Hammerstein models are a simplification of this "sandwich" approach. In a cascade of Hammerstein system [13], each branch is composed of one nonlinear static polynomial element followed by a linear one $h_{n}(t)$ as shown in Fig. 1(a). The relation between the input $e(t)$ and the output $s(t)$ of such a system is given by Eq. (1) where " $(*)$ " denotes the convolution 


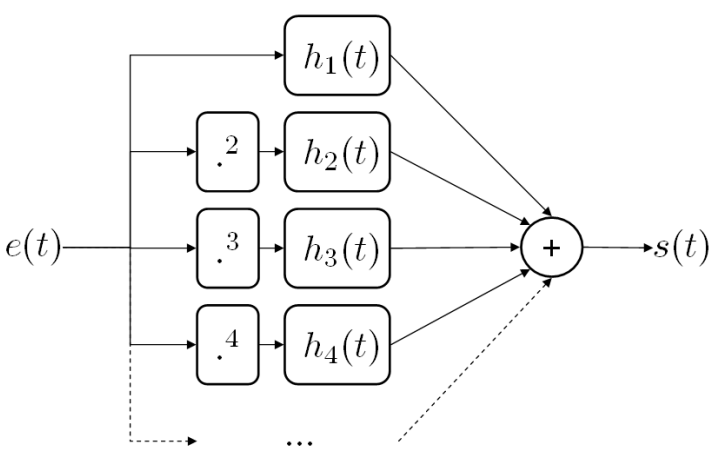

(a)

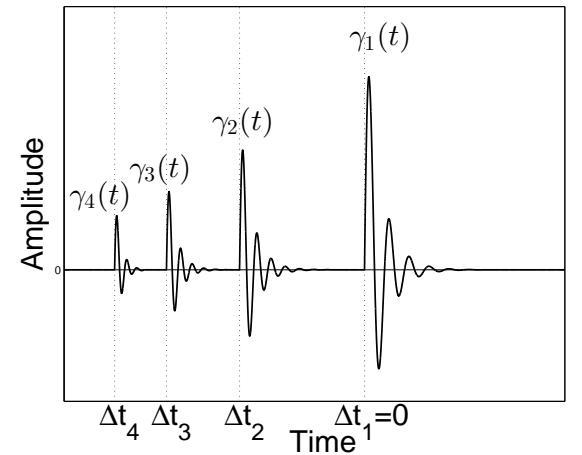

(b)

Figure 1: (a) Cascade of Hammerstein model and (b) temporal separation after deconvolution.

operator.

$$
s(t)=\sum_{n=1}^{N}\left(h_{n} * e^{n}\right)(t)
$$

92

It can easily be shown from Eq. (1) that cascades of Hammerstein models correspond to Volterra models having diagonal Kernels in the temporal domain [15]. Thus, cascades of Hammerstein models represent a subclass of all the nonlinear "analytical" systems described by Volterra models, and are thus rather general nonlinear models. Furthermore, any cascade of Hammerstein models is fully represented by its kernels $\left\{h_{n}(t)\right\}_{n \in\{1 \ldots N\}}$, which are only a set of linear filters. This model is thus also quite simple to use and intuitive to understand.

\subsection{Exponential sine sweeps}

Estimating each kernel $h_{n}(t)$ of a cascade of Hammerstein models is not a straightforward task. An simple estimation method that has been proposed 
previously by the authors [15] for this purpose and that is the basis of the damage detection procedure is briefly recalled here.

To experimentally cover the frequency range over which the system under study has to be identified, cosines with time-varying frequencies are commonly used. Indeed, if $e(t)=\cos [\phi(t)]$ is the input of the cascade of Hammerstein models, the output of the nonlinear block $e^{n}(t)$, see Fig. 1(a), can be rewritten using Chebyshev polynomials as in Eq. (2). Details of the computation of the Chebyshev matrix $C=\left\{c_{n, k}\right\}$ are provided in [15].

$$
\forall n \in[1 . . N] \quad e^{n}(t)=\cos ^{n}[\phi(t)]=\sum_{k=0}^{n} c_{n, k} \cos [k \phi(t)]
$$

When the instantaneous frequency of $e(t)$ is increasing exponentially from $f_{1}$ to $f_{2}$ in a time interval $T$, this signal is called an "Exponential Sine Sweep". It can be shown in $[14,15]$, that by choosing $T_{m}=\left(2 m-\frac{1}{2}\right) \frac{\ln \left(f_{2} / f_{1}\right)}{2 f_{1}}$ with $m \in \mathbb{N}^{*}$, one obtains:

$$
\forall k \in \mathbb{N}^{*} \quad \cos [k \phi(t)]=\cos \left[\phi\left(t+\Delta t_{k}\right)\right] \quad \text { with } \quad \Delta t_{k}=\frac{T_{m} \ln (k)}{\ln \left(f_{2} / f_{1}\right)}
$$

Eq. (3) is another expression of the $k^{\text {th }}$ term in the linearization presented in Eq. (2). In summary, for any exponential sine sweep of duration $T_{m}$, multiplying the phase by a factor $k$ yields to the same signal, advanced in time by $\Delta t_{k}$.

\subsection{Kernel recovery in the temporal domain}

If an exponential sine sweep is presented at the input of a cascade of Hammerstein models, we obtain by combining Eq. (3) and Eq. (1) the following relation: 


$$
s(t)=\sum_{n=1}^{N}\left(\gamma_{n} * e\right)\left(t+\Delta t_{n}\right) \quad \text { with } \quad \gamma_{n}(t)=\sum_{k=1}^{n} C(k, n) h_{k}(t)
$$

where $\gamma_{n}(t)$ corresponds to the contribution of the different kernels to the $n^{\text {th }}$ harmonic.

In order to separately identify each kernel $h_{n}(t)$ of the cascade of Hammerstein models, a signal $y(t)$ operating as the inverse of the input signal $e(t)$ in the convolution sense, is needed. The Fourier transform $Y(f)$ of the inverse filter $y(t)$ can be built by means of Eq. (5):

$$
Y(f)=\frac{1}{E(f)} \simeq \frac{\bar{E}(f)}{|E(f)|^{2}+\epsilon(f)}
$$

where $E(f)$ and $\bar{E}(f)$ are respectively the Fourier transform of $e(t)$ and its complex conjugate, and $\epsilon(f)$ is a frequency-dependent real parameter chosen to be 0 in the bandwidth of interest and to have a large value outside, with a continuous transition between the two domains, see [15].

After convolving the output of the cascade of Hammerstein models $s(t)$ given in Eq. (4) with $y(t)$, one obtains Eq. (6), also illustrated in Fig. 1(b):

$$
(y * s)(t)=\sum_{n=1}^{N} \gamma_{n}\left(t+\Delta t_{n}\right)
$$

Because $\Delta t_{n} \propto \ln (n)$ and $f_{2}>f_{1}$, the higher the order of non-linearity $n$, the more advanced is the corresponding $\gamma_{n}(t)$, see Fig. 1(b). Thus, if $T_{m}$ is chosen long enough, the different $\gamma_{n}(t)$ do not overlap in time and can be separated by simply windowing them in the time domain. Using Eq. (7), the family $\left\{h_{n}(t)\right\}_{n \in[1, N]}$ of the kernels of the cascade of Hammerstein models under study can then be fully extracted. 


$$
\left(\begin{array}{c}
h_{1}(t) \\
\vdots \\
h_{N}(t)
\end{array}\right)=\tilde{C}^{T}\left(\begin{array}{c}
\gamma_{1}(t) \\
\vdots \\
\gamma_{N}(t)
\end{array}\right)
$$

In Eq. (7), $C^{T}$ stands for the transpose of the Chebyshev matrix $C$, and $\tilde{C}$ represents $C$, from which the first column and the first row have been removed.

It can be noticed here that the proposed method is not fully nonparametric. Indeed, one parameter, $N$ the order of nonlinearity up to which the nonlinear model has to be estimated, is still to be chosen. Its choice mainly depends on the noise conditions and on the length of the input exponential sine sweep [15].

\section{Novelty damage indexes}

In the case of a structure with distributed actuators and sensors, we can consider several configurations to perform damage monitoring. As here an active SHM approach has been retained, measurements of one sensor can be used by defining a path over the structure that leads to a single-input singleoutput (SISO) system. However, the measurements of all sensors can also be used simultaneously, thus defining in that case a single-input multi-output (SIMO) system. The two novelty damage indexes proposed in the following correspond to these two configurations.

\subsection{Decomposition of the output signal into linear and nonlinear parts}

By rephrasing Eq. (1) which expresses the output of the cascade of Hammerstein models $s(t)$ as a function of the input signal $e(t)$ and of the Ham- 
merstein kernels $\left\{h_{n}(t)\right\}_{n \in[1, N]}$, it is possible to decompose the output of the cascade of Hammerstein models as follows:

$$
s(t)=\left(h_{1} * e\right)(t)+\sum_{n=2}^{N}\left(h_{n} * e^{n}\right)(t)=s^{\mathrm{L}}(t)+s^{\mathrm{NL}}(t)
$$

where $s^{\mathrm{L}}(t)=\left(h_{1} * e\right)(t)$ stand for the linear and $s^{\mathrm{NL}}(t)=\sum_{n=2}^{N}\left(h_{n} * e^{n}\right)(t)$ the nonlinear parts of the output signal $s(t)$.

As the input signal $e(t)$ is known and as the Hammerstein kernels $\left\{h_{n}(t)\right\}_{n \in[1, N]}$ have been estimated previously, those linear and nonlinear parts of the output signal are then easily evaluated and can be used to build damage indexes.

\section{2. $D I_{1}$ : Ratio of the nonlinear energy to the linear energy}

In the single-input, single-output case (SISO), there is only one input $e(t)$ and one output $s(t)$. Taking advantage of Eq. (8), we propose a damage index (DI) that is defined as the ratio of the energy contained in the nonlinear part of the output of the cascade of Hammerstein models versus the energy contained in the linear part of the output of the cascade of Hammerstein models. By denoting $S^{\mathrm{L}}(f)$ and $S^{\mathrm{NL}}(f)$ the Fourier transform of $s^{\mathrm{L}}(t)$ and $s^{\mathrm{NL}}(t)$, we propose a damage index defined as follow:

$$
\mathrm{DI}_{1}=\frac{\int_{f_{1}}^{f 2}\left|S^{\mathrm{NL}}(f)\right|^{2} d f}{\int_{f_{1}}^{f 2}\left|S^{\mathrm{L}}(f)\right|^{2} d f}
$$

where $f_{1}$ and $f_{2}$ have been defined earlier in Sec. 2.2.

In a given composite structure, as the nonlinear damage (impact, delamination, or crack) becomes more severe, it is expected to contribute more and more to the nonlinear part of the output $s^{\mathrm{NL}}(t)$. As a consequence, $\mathrm{DI}_{1}$ is 
expected to be sensitive to the presence of the damage, but also to its extent. This will be demonstrated numerically in Sec. 4.3 and experimentally in Sec. 5.

\section{3. $\mathrm{DI}_{2}:$ Angle between nonlinear subspaces}

In the single-input, multiple-output framework (SIMO), there is still one input $e(t)$ but now $J$ outputs $\left\{s_{j}(t)\right\}_{j \in[1 . . J]}$. Taking advantage of Eq. (8),

it is still possible to decompose each output $s_{j}(t)$ into its linear $s_{j}^{\mathrm{L}}(t)$ and nonlinear $s_{j}^{\mathrm{NL}}(t)$ parts. Following previous work by the authors [17], the idea is then to monitor the subspaces spanned by the nonlinear parts of each outputs set.

In a discrete-time matrix form, let $\boldsymbol{s}^{\mathrm{NL}} \in \mathbb{R}^{U \times J}$ be the nonlinear parts of the $J$ output signals having each a length of $U$ samples. Let $\boldsymbol{A}^{\mathrm{NL}} \in \mathbb{R}^{U \times J}$ be the separating matrix of $s^{\mathrm{NL}}$. This matrix is obtained from a principal component analysis technique [18] and is defined as follows:

$$
\boldsymbol{A}^{\mathrm{NL}}=\boldsymbol{\Lambda}_{\boldsymbol{s}^{\mathrm{NL}}}^{-\frac{1}{2}} \times\left(\boldsymbol{P}^{\mathrm{NL}}\right)^{\mathrm{T}}
$$

194 where $\boldsymbol{P}^{\mathrm{NL}}=\left[\underline{\boldsymbol{p}}_{1}^{\mathrm{NL}}, \ldots, \underline{\boldsymbol{p}}_{J}^{\mathrm{NL}}\right]$ is the matrix of eigenvectors of $\boldsymbol{s}^{\mathrm{NL}}$ and $\Lambda_{s^{\mathrm{NL}}}$ is the diagonal matrix of eigenvalues of $\boldsymbol{s}^{\mathrm{NL}}$. If the reduction using singular value decomposition (SVD) is possible [19], the separating matrix can then be rewritten as follows: 


$$
\begin{aligned}
\boldsymbol{A}^{\mathrm{NL}} & =\boldsymbol{I}_{J \times J} \times \boldsymbol{\Gamma}^{\mathrm{NL}} \times\left(\boldsymbol{V}^{\mathrm{NL}}\right)^{\mathrm{T}} \\
& =\left[\begin{array}{ll}
\boldsymbol{I}_{J \times J_{p}} & \boldsymbol{I}_{J \times\left(J-J_{p}\right)}
\end{array}\right]\left[\begin{array}{cc}
\boldsymbol{\Gamma}_{1}^{\mathrm{NL}} & \mathbf{0} \\
\mathbf{0} & \boldsymbol{\Gamma}_{2}^{\mathrm{NL}}
\end{array}\right]\left[\begin{array}{cc}
\boldsymbol{V}_{1}^{\mathrm{NL}} & \boldsymbol{V}_{2}^{\mathrm{NL}}
\end{array}\right]^{\mathrm{T}} \\
& =\boldsymbol{A}_{1}^{\mathrm{NL}}+\boldsymbol{A}_{2}^{\mathrm{NL}}
\end{aligned}
$$

where $\boldsymbol{\Gamma}_{1}^{\mathrm{NL}}=\operatorname{diag}\left(\sigma_{1}, \ldots, \sigma_{J_{p}}\right), \boldsymbol{V}_{1}^{\mathrm{NL}}=\left[\underline{\boldsymbol{v}}_{1_{1}}^{\mathrm{NL}}, \ldots, \underline{\boldsymbol{v}}_{1_{J_{p}}}^{\mathrm{NL}}\right] \in \mathbb{R}^{U \times J_{p}}$ and $\boldsymbol{A}_{1}^{\mathrm{NL}} \in \mathbb{R}^{U \times J_{p}}$ are respectively the matrix of singular values, the matrix of right singular vectors, and the separating matrix associated to the principal subspace of $\boldsymbol{s}^{\mathrm{NL}} \cdot \boldsymbol{\Gamma}_{2}^{\mathrm{L}}=\operatorname{diag}\left(\sigma_{J_{p}+1}, \ldots, \sigma_{J}\right), \boldsymbol{V}_{2}^{\mathrm{NL}}=\left[\underline{\boldsymbol{v}}_{1_{J_{p}+1}}^{\mathrm{NL}}, \ldots, \underline{\boldsymbol{v}}_{1_{J}}^{\mathrm{NL}}\right] \in$ $\mathbb{R}^{U \times\left(J-J_{p}\right)}$ and $\boldsymbol{A}_{2}^{\mathrm{NL}} \in \mathbb{R}^{U \times\left(J-J_{p}\right)}$ are respectively the matrix of singular values, the matrix of right singular vectors, and the separating matrix associated to the residual subspace of $\boldsymbol{s}^{\mathrm{NL}}$.

Let $\boldsymbol{A}_{1}^{\mathrm{NL}}$ and $\tilde{\boldsymbol{A}}_{1}^{\mathrm{NL}}$ be two matrices built as described previously from measurements in a healthy state and in an unknown state. Let's $R\left\{\left(\boldsymbol{A}_{1}^{\mathrm{NL}}\right)^{\mathrm{T}}\right\}$ and $R\left\{\left(\tilde{\boldsymbol{A}}_{1}^{\mathrm{NL}}\right)^{\mathrm{T}}\right\}$ be the range subspaces of matrices $\left(\boldsymbol{A}_{1}^{\mathrm{NL}}\right)^{\mathrm{T}}$ and $\left(\tilde{\boldsymbol{A}}_{1}^{\mathrm{NL}}\right)^{\mathrm{T}}$, and $\boldsymbol{P}_{R\left\{\left(\boldsymbol{A}_{1}^{\mathrm{NL}}\right)^{\mathrm{T}}\right\}}$ and $\boldsymbol{P}_{R\left\{\left(\tilde{\boldsymbol{A}}_{1}^{\mathrm{L}}\right)^{\mathrm{T}}\right\}}$ the orthogonal projections on these range subspaces obtained though SVD (see [17] for details). We then denote $\underline{\phi}\left[R\left\{\left(\boldsymbol{A}_{1}^{\mathrm{NL}}\right)^{\mathrm{T}}\right\}, R\left\{\left(\tilde{\boldsymbol{A}}_{1}^{\mathrm{NL}}\right)^{\mathrm{T}}\right\}\right]$ the principal angle vectors between the range subspaces $R\left\{\left(\boldsymbol{A}_{1}^{\mathrm{NL}}\right)^{\mathrm{T}}\right\}$ and $R\left\{\left(\tilde{\boldsymbol{A}}_{1}^{\mathrm{L}}\right)^{\mathrm{T}}\right\}$. Using the SVD tool, the Euclidean norm of the sinus of this angle is defined as follow [20]:

$$
\begin{aligned}
\left\|\sin \left(\underline{\phi}\left[R\left\{\left(\boldsymbol{A}_{1}^{\mathrm{NL}}\right)^{\mathrm{T}}\right\}, R\left\{\left(\tilde{\boldsymbol{A}}_{1}^{\mathrm{L}}\right)^{\mathrm{T}}\right\}\right]\right)\right\|_{2} & =\left\|\boldsymbol{P}_{R\left\{\left(\boldsymbol{A}_{1}^{\mathrm{NL}}\right)^{\mathrm{T}}\right\}^{\perp}} \times \boldsymbol{P}_{R\left\{\left(\tilde{\boldsymbol{A}}_{1}^{\mathrm{NL}}\right)^{\mathrm{T}}\right\}}\right\|_{2}(12) \\
& =\left\|\left(\boldsymbol{I}_{J_{p} \times J_{p}}-\boldsymbol{P}_{R\left\{\left(\boldsymbol{A}_{1}^{\mathrm{NL}}\right)^{\mathrm{T}}\right\}}\right) \times \boldsymbol{P}_{R\left\{\left(\tilde{\boldsymbol{A}}_{1}^{\mathrm{NL}}\right)^{\mathrm{T}}\right\}}\right\|_{2}
\end{aligned}
$$


We then propose to define a damage index as:

$$
\mathrm{DI}_{2}=\frac{\left\|\sin \left(\underline{\phi}\left[R\left\{\left(\boldsymbol{A}_{1}^{\mathrm{NL}}\right)^{\mathrm{T}}\right\}, R\left\{\left(\tilde{\boldsymbol{A}}_{1}^{\mathrm{NL}}\right)^{\mathrm{T}}\right\}\right]\right)\right\|_{2}}{J_{p}}
$$

where $J_{p}$ is the number of principal components retained in the principal subspaces. This damage index can be interpreted as the angle between the subspaces described by the nonlinear parts of the outputs in the healthy state and in the unknown state.

In a given composite structure, as the nonlinear damage becomes more severe, it is expected to contribute more and more to the nonlinear parts of the different outputs $\left\{s_{j}(t)\right\}_{j \in[1 . . J]}$ and then to increase the angle between the associated principal subspaces. As a consequence, $\mathrm{DI}_{2}$ is expected to be sensitive to the presence of the damage, but also to its extent. This will be demonstrated numerically in Sec. 4.4 and experimentally in Sec. 5.

\section{Simulation results}

\subsection{Simulated systems}

In order to validate the proposed approach and the associated novelty damage indexes, numerical simulations have been carried out for single-input, single-output (SISO) and single-input, multi-output (SIMO) systems. The systems that have been chosen are simple one degree of freedom and five degrees of freedom spring-mass-damper (SMD) systems and are shown in Fig. 2.

The damage in those systems has been introduced by means of a bilinear stiffness $k[x(t)]$ as a very easy way to simulate a breathing crack. Such cracks 


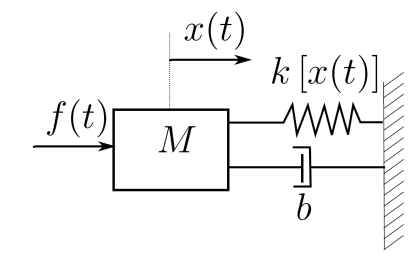

(a)

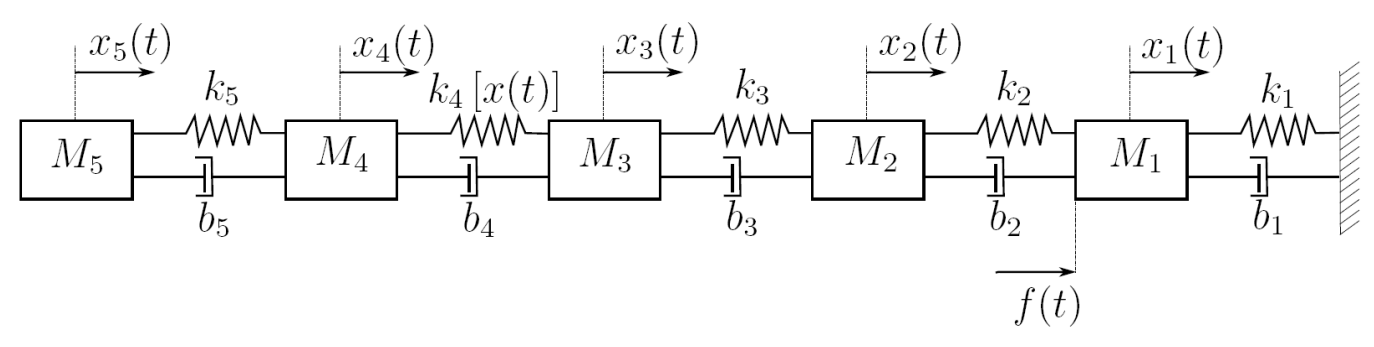

(b)

Figure 2: (a) Simulated single degree of freedom SISO system and (b) simulated five degrees of freedom SIMO system.

have a lower stiffness when the crack is open than when the crack is closed. Thus, the bilinear stiffness is defined as follows:

$$
k[x(t)]=\left\{\begin{array}{ccc}
k^{I} & \text { if } & x(t)<0 \\
(1-\alpha) k^{I} & \text { if } & x(t)>0
\end{array}\right.
$$

In this definition, $k^{I}$ denotes the linear stiffness of the original undamaged system and the damage-parameter is the coefficient $\alpha$. If $\alpha=0$, the stiffness is fully linear and the system is healthy. If $\alpha=1$, the stiffness when the crack is open is null and thus, the system if fully damaged.

The chosen SISO system is a SMD system where the input is the force $f(t)$ applied to the mass $M$ and the output is the displacement $x(t)$ of the mass $M$, as shown in Fig. 2(a). For this system $M=1 \mathrm{~kg}, b=2 \mathrm{Ns} / \mathrm{m}$ and 
$k^{I}=20000 \mathrm{~N} / \mathrm{m}$.

The chosen SIMO system is a serie of five SMD systems where the input is the force $f(t)$ applied to the mass $M_{1}$ and the outputs are the displacements $\left\{x_{1}(t), \ldots, x_{5}(t)\right\}$ of the masses $\left\{M_{1}, \ldots, M_{5}\right\}$ as shown in Fig. 2(b). The damage is introduced by means of the bilinear stiffness $k_{4}[x(t)]$ as defined by Eq. (14). For this system $M_{1}=M_{2}=M_{3}=M_{4}=M_{5}=1 \mathrm{~kg}, b_{1}=b_{2}=b_{3}=$ $b_{4}=b_{5}=2 \mathrm{Ns} / \mathrm{m}, k_{1}=k_{2}=k_{3}=k_{5}=20000 \mathrm{~N} / \mathrm{m}$, and $k_{4}^{I}=20000 \mathrm{~N} / \mathrm{m}$.

\subsection{Input signal}

In order to estimate the linear and nonlinear parts in the ouptut of this system, an input signal has been designed as described in Sec. 2.2. The start and stop frequencies have been chosen as $f_{1}=2.25 \mathrm{~Hz}$ and $f_{2}=225 \mathrm{~Hz}$, knowing that $f_{r}=25 \mathrm{~Hz}$ is the resonance frequency of both undamaged systems. The sweep duration has been chosen as $T=8.86$ seconds for the SISO system and $T=88.6$ seconds for the SIMO system both with an input amplitude of $E=0.1 \mathrm{~N}$. The response of this system to this input signal has been simulated using Simulink ${ }^{\mathrm{TM}}$ with a fixed-step Runge-Kutta algorithm running at $f_{s}=563 \mathrm{~Hz}$. A zero-mean Gaussian white noise has been added to the input of the simulation in order to simulate environmental noise. It is assumed that environmental noise is larger than measurement noise, and thus no noise has been added to the output of the simulation. To illustrate the robustness of the proposed DIs to noise, the noise variance has been chosen as a function of the root-mean-square power of the input signal in order to have a signal to noise ratio (SNR) of 60 or $30 \mathrm{~dB}$ (i.e. a noise with a standard deviation of $6 \times 10^{-5} \mathrm{~N}$ or $\left.2 \times 10^{-3} \mathrm{~N}\right)$. Simulation have been carried out for values of $\alpha$ ranging from 0 (healthy state) to 0.45 (half-damaged state) 
(a)

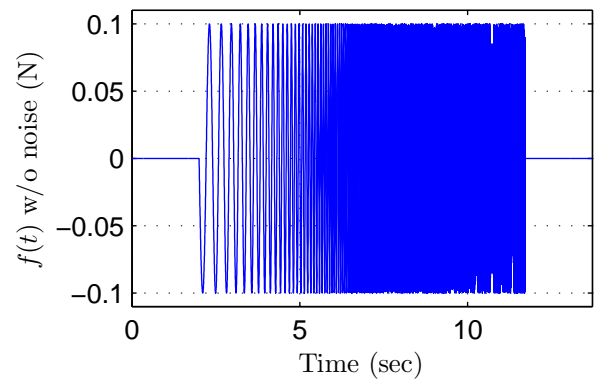

(b)

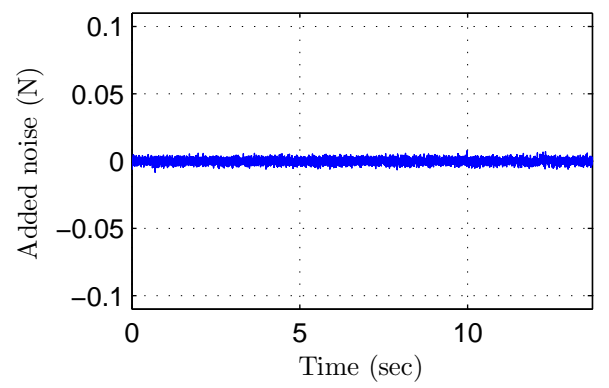

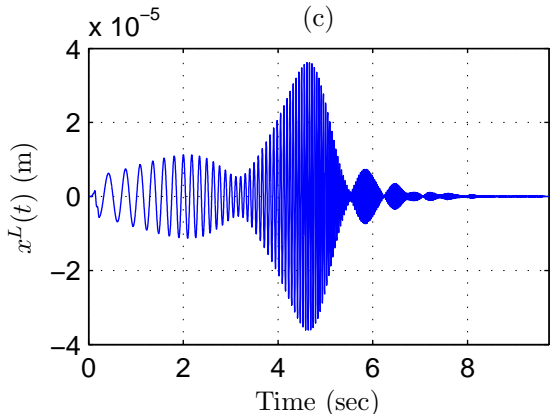

(d)

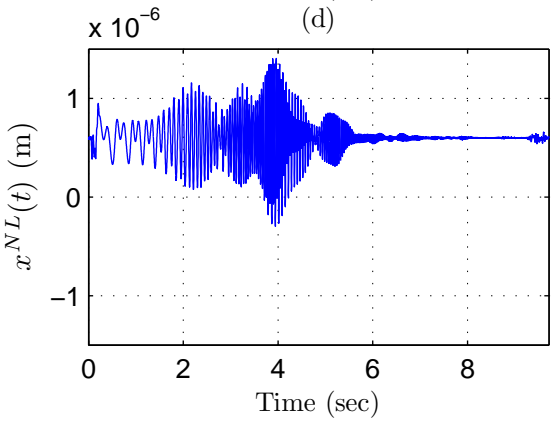

Figure 3: (a) Input sweep without noise and (b) added noise for a SNR of $30 \mathrm{~dB}$. Estimated (c) linear and $(\mathrm{d})$ nonlinear parts of the output signal $x(t)$.

272

The noise-free input signal, the added noise, and the estimated linear $x^{L}(t)$ and nonlinear $x^{N L}(t)$ parts of the output signal $x(t)$ of the system of Fig. 2(a) are shown on Fig. 3. From this figure, it can seen that a SNR of $30 \mathrm{~dB}$ implies the addition of a relatively large amount of noise to the input signal. Furthermore, by analyzing the estimated linear part of the ouptut 
signal $x^{L}(t)$, it can be seen that, as expected, the chosen nonlinear system basically acts as a resonant filter. Finally, it can be seen that the system under study is effectively nonlinear as a non-null nonlinear part $x^{N L}(t)$ in the output signal is being estimated by the previously described procedure.
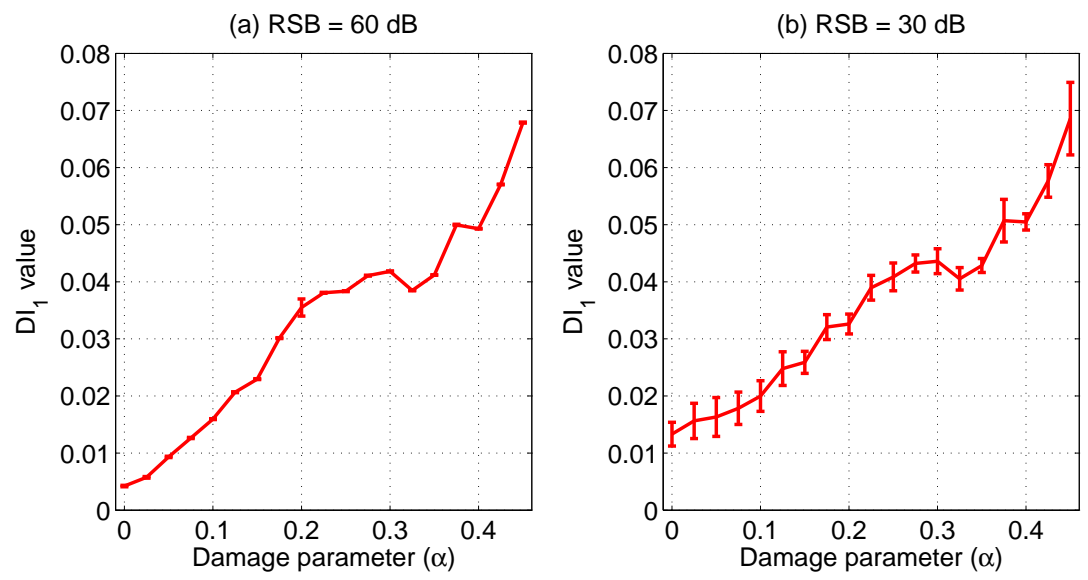

Figure 4: Average and standard deviation of the $\mathrm{DI}_{1}$ values for different values of the damage parameter $\alpha$ with (a) $\mathrm{SNR}=60 \mathrm{~dB}$ and (b) $\mathrm{SNR}=30 \mathrm{~dB}$.

In Fig. 4 the averages and standard deviations over the 30 trials of the $\mathrm{DI}_{1}$ values for the different values of the damage parameter $\alpha$ and with $\mathrm{SNR}=60 \mathrm{~dB}$ and $\mathrm{SNR}=30 \mathrm{~dB}$ are shown. First of all, it can be seen that the damage index $\mathrm{DI}_{1}$ increases in both cases almost monotically with the damage parameter $\alpha$. Moreover, even when the noise power is relatively large (see the curve for $\mathrm{SNR}=30 \mathrm{~dB}$ ) the standard deviations remain small around the average values. Finally, for a value of the damage parameter $\alpha=0$ (i.e. in the linear case), the $\mathrm{DI}_{1}$ value should be zero and is found different from zero. This thus means that a part of the noise is here interpreted by the estimation process as a nonlinear part of the output. We can thus 


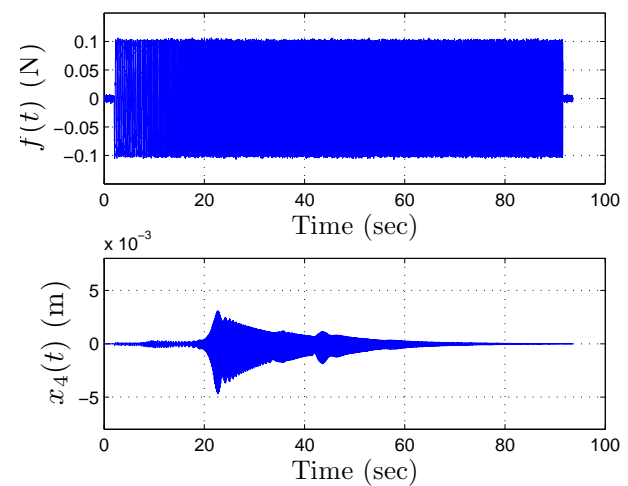
robustness to noise.

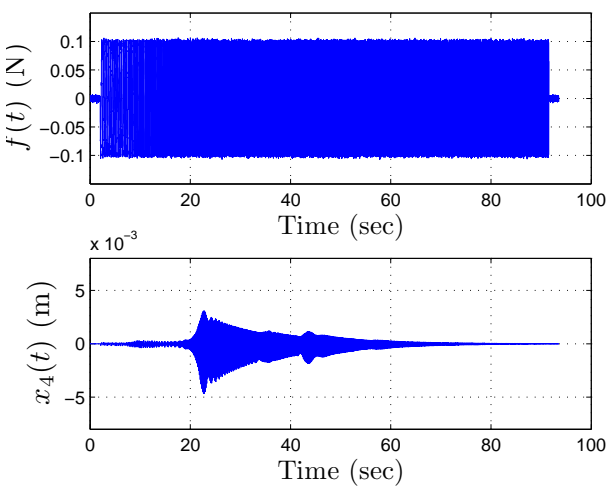

(a)

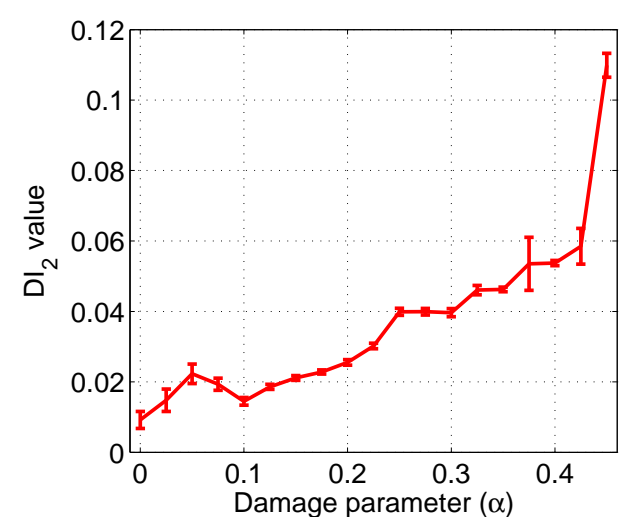

(b)

Figure 5: (a) Input signal $f(t)$ (top) and output signal $x_{4}(t)$ for $\alpha=0.45$ (bottom). (b) Average and standard deviation of the $\mathrm{DI}_{2}$ values for different values of the damage parameter $\alpha$ and for a SNR of $30 \mathrm{~dB}$.

conclude from that simulation that the damage index $\mathrm{DI}_{1}$ is able to detect and to quantify the amount of damage in the nonlinear system with a high

\subsection{Damage detection using $\mathrm{DI}_{2}$ for the SIMO system}


of the damage parameter $\alpha$ and for a SNR of $30 \mathrm{~dB}$ are shown. First of all, it can be seen that again the damage index $\mathrm{DI}_{2}$ increases almost monotically with the damage parameter $\alpha$. Moreover, even if the noise power is relatively large $(\mathrm{SNR}=30 \mathrm{~dB})$ the standard deviations still remain very small around the average values. The curves for a SNR of $60 \mathrm{~dB}$ are not shown here as they are very similar to the one for a SNR of $30 \mathrm{~dB}$ but with lower standard deviations. We can thus conclude from that simulation that the damage index $\mathrm{DI}_{2}$ is here also able to detect and to quantify the amount of damage in the nonlinear system with a high robustness to noise.

\section{Experimental results}

\subsection{Plate specimens}

The two composite plates employed in this study consist of a piece of aircraft composite fuselage. The dimensions of these structures are $(400 \times$ $300 \times 2 \mathrm{~mm}^{3}$ ). They are both made up of 16 layers Carbone epoxy material. The layer sequences are: $\left(0^{\circ}, 45^{\circ},-45^{\circ}, 90^{\circ}, 90^{\circ},-45^{\circ}, 45^{\circ}, 0^{\circ}\right)$. An optimal placement of ten PZ29 piezoceramic patches with dimensions $(30 \times 20 \times$ $0.2 \mathrm{~mm}^{3}$ ) has been achieved on these two structures using the controllability and observability gramians [21]. The composite plate shown in Fig. 6(a) was used as the baseline for damage detection. Fig. 6(b) shows the second composite plate, manufactured from the same material and layer sequences, having the same dimensions and PZT number and placement as the first one. However, in this plate, an calibrated impact damage with a $5 \mathrm{~mm}$ diameter was produced by projecting on the center of the plate a steel ball at a high and controlled velocity. This composite plate will be used as a damaged plate 
example.

\subsection{Data acquisition and Hammerstein Kernels estimation}

The input excitation and the data acquisition were performed using a voltage amplifier (TREK MODEL 601C) and charge amplifiers (type 5011B). This excitation was applied sequentially to nine PZT elements and consists of an exponential sine sweep signal with $f_{1}=100 \mathrm{~Hz}, f_{2}=30 \mathrm{kHz}, T=3.2588 \mathrm{~s}$ and an amplitude of $10 \mathrm{~V}$ (see Sec. 2). Using a real time prototype system dSPACE, temporal signals were acquired with a sampling frequency $f_{s}=$ $100 \mathrm{kHz}$ from nine channels: one corresponding to the excitation applied to a given PZT actuator, and the eight others corresponding to the measurements collected by the PZT sensors. Under those conditions, the SNR is found to be approximately of $60 \mathrm{~dB}$.

A first database has been built, by collecting 10 times in the healthy and damaged states the signals for all the paths starting from the PZT element number 7 , located near the center of the plate, close to the damage. The aim of this database is to quantify the environmental variability existing for a given path by computing the damage index $\mathrm{DI}_{1}$ mean and standard deviation over the 10 trials and to infer a detection threshold for $\mathrm{DI}_{2}$ defined by Eq. (13). Another database has been built by collecting in each state (healthy or damaged) the signals for the $9 \times 8=72$ paths existing between all pairs of PZT elements. The objective of this database is to illustrate the ability of both DIs to detect damages among the different paths and actuators that are considered. For both databases, the Hammerstein Kernels have been estimated using the method described in Sec. 2 up to an order of nonlinearity $N=8$. This choice has been done with respects to noise conditions and to 
the length of the input exponential sine sweep [15].

\subsection{Sensibility to environmental noise and to damage for one actuator}

To assess the sensibility of the $\mathrm{DI}_{1}$ defined in Eq.(9) to the presence of environmental noise, $\mathrm{DI}_{1}$ values have been computed for all the repetitions for the healthy and damaged plates, as described in Sec. 5.2. In Fig. 7(a), the mean and standard deviation of the $\mathrm{DI}_{1}$ values computed over the 10 repetitions for each path are shown for both states. From this figure, we can see that even in the healthy state, the $\mathrm{DI}_{1}$ values are around 0.77 . This means that there is a non-negligible part of the energy in the nonlinear part of the output and thus that the system under study is nonlinear in its healthy state. This illustrates the fact that the proposed method can handle systems that are nonlinear in their healthy state. From that figure, it can also be seen that the variations caused by environmental noise on $\mathrm{DI}_{1}$ values remains relatively low and that the $\mathrm{DI}_{1}$ values for the damaged case are well above the $\mathrm{DI}_{1}$ values for the healthy state. As such, we can conclude that experimentally the proposed $\mathrm{DI}_{1}$ is not much sensitive to environmental noise and is effectively sensitive to the presence of the damage.

The damage index $\mathrm{DI}_{2}$ defined by Eq. (13) is comparing the nonlinear subspaces spanned by a reference state and by an unknown state. As so, this DI is relative by nature and a decision threshold needs to be defined in order to decide whether or not there is presence of a damage. To do so, we decided here to proceed experimentally by using the 10 repetitions for PZT 7 in the healthy state. The first repetition has been chosen as the reference state, and the nine others as unknown (but healthy) states. The $\mathrm{DI}_{2}$ values obtained by comparing these unknown (but healthy) states to the reference 
one are plotted on Fig. 7(b). It can be seen that the environmental noise does not cause large changes to these $\mathrm{DI}_{2}$ values. On the basis of these values, a decision threshold has then been defined as ten times the maximum value obtained previously. The factor ten is arbitrarily chosen but is thought here to be sufficiently large for reasonable decision making. This decision threshold is also plotted on Fig. 7(b). Now, the impact of environmental noise in the damaged case can be assessed by comparing, for each trial the $\mathrm{DI}_{2}$ value obtained by comparing the healthy and damaged states. As shown in Fig. 7(b), for each trial, the obtained $\mathrm{DI}_{2}$ values are not so influenced by noise and are always above the decision threshold value. Thus, we can say that the $\mathrm{DI}_{2}$ defined by Eq. (13) is not very sensitive to noise and appears to be sensitive to the presence of the damage for this actuator.

\subsection{Sensibility to the presence of the damage for all the actuators}

To assess the sensibility of the $\mathrm{DI}_{1}$ defined in Eq. (9) to the presence of the damage for different actuators and paths over the plate, $\mathrm{DI}_{1}$ values have been computed for each of the 72 paths measured on the healthy and damaged plates (as described in Sec. 5.2). For sake of brevity, $\mathrm{DI}_{1}$ values are presented here in a synthetic manner actuator by actuator. Fig. 8(a) depicts the mean and standard deviation of the $\mathrm{DI}_{1}$ values computed for all the paths starting from each of the nine PZT elements used as actuators for both the healthy and damaged states. From Fig. 8(a), it is clear that the damage introduced in the plate generates nonlinearities and that the $\mathrm{DI}_{1}$ as defined in Eq. (9) is sensitive to the presence of this damage. Indeed, for all the actuators, the mean $\mathrm{DI}_{1}$ values computed for all the paths starting from a given actuator are higher for the damaged state than for the healthy one. 
To assess the sensibility of the $\mathrm{DI}_{2}$ defined in Eq. (13) to the presence of the damage for different actuators and paths over the plate, $\mathrm{DI}_{2}$ values have been computed for each of the 9 actuators using measurements from the healthy and damaged plates (as described in Sec. 5.2). These damage index values are compared in Fig. 8(b) to the detection threshold defined in the previous section. From Fig. 8(b), it is clear that the damage introduced in the plate generates nonlinearities and that the $\mathrm{DI}_{2}$ as defined in Eq. (13) is sensitive to the presence of this damage. Indeed, for all the actuators, the obtained $\mathrm{DI}_{2}$ values are higher for the damaged state than the chosen decision threshold.

\section{Conclusion}

In many cases, damages that appear on complex structures (such as cracks, impacts, or delaminations) can result in nonlinear dynamical responses that may be used for damage detection. Furthermore, complex structures often exhibit a nonlinear behavior even in their healthy states. A robust and reliable SHM system must then be able to deal with nonlinear damages, and to distinguish between their effects and inherent nonlinearities in healthy structures. The first problem to be addressed is that the nonlinear models already in use are never general enough to encompass all the structure encountered in real life. The second problem is that the currently developed nonlinear models are not adequate for practical use of SHM systems. The work presented here attempts to face these two problems on the basis of a simple, but rather general, nonlinear model estimated by means of standard signal processing tools. This approach is based on the assumption that the 
structure under study can be modeled as a cascade of Hammerstein models. The Exponential Sine Sweep Method, previously developed and validated by the authors for different purposes, is then used to estimate the different kernels of the model. Exponential sine sweeps are a class of sine sweeps that allow estimating a model in a wide frequency band from only one measurement. Two damage indexes are then build on the basis of this estimated model. The first one reflects the ratio of the energy contained in the nonlinear part of the output versus the energy contained in its linear part and is specially suited for single-input single-output (SISO) systems. The second one is the angle between the subspaces described by the nonlinear parts of two set of outputs after a principal component analysis. This one is specially suited for single-input multi-output (SIMO) systems. As a first step toward the use of this method for SHM, the sensitivity of the proposed DIs to the presence of damages as well as their robustness to noise are assessed numerically on SISO and SIMO systems and experimentally on two actual composite plates with surface-mounted PZT-elements (one healthy and one damaged).

The work presented here is however only a first step toward a larger use of this method in SHM. Indeed, it has be shown here that the proposed DIs are effectively sensitive to the presence of a non-linear damage and that they can potentially be helpful to quantify its extent. However, this approach can also be cast in the context of a statistical pattern recognition problem. Then, the DIs defined here, or other defined on the basis of the estimated model, can be used to train expert systems that are able to distinguish between different kind of damages [22]. 


\section{References}

[1] C. R. Farrar, K. Worden, M. D. Todd, G. Park, J. Nichols, D. E. Adams, M. T. Bement, M. T. Farinholt, Nonlinear system identification for damage detection, Tech. rep., Los Alamos National Laboratory (2007).

[2] K. Worden, C. R. Farrar, J. Haywood, M. Todd, A review of nonlinear dynamics applications to structural health monitoring, Structural Control \& Health Monitoring 15 (4) (2008) 540-567.

[3] J. M. Nichols, M. D. Todd, Encyclopedia of Structural Health Monitoring, John Wiley \& Sons, Ltd, 2009, Ch. Nonlinear Features for SHM Applications.

[4] G. Kerschen, K. Worden, A. F. Vakakis, J. C. Golinval, Past, present and future of nonlinear system identification in structural dynamics, Mechanical Systems and Signal Processing 20 (3) (2006) 505-592.

[5] T. J. Johnson, D. E. Adams, Transmissibility as a differential indicator of structural damage, Journal of Vibration and Acoustics-transactions of the Asme 124 (4) (2002) 634-641.

[6] M. Haroon, D. E. Adams, Time and frequency domain nonlinear system characterization for mechanical fault identification, Nonlinear Dynamics 50 (3) (2007) 387-408.

[7] Z. Q. Lang, Z. K. Peng, A novel approach for nonlinearity detection in vibrating systems, Journal of Sound and Vibration 314 (3-5) (2008) 603-615. 
[8] Z. Q. Lang, G. Park, C. R. Farrar, M. D. Todd, Z. Mao, L. Zhao, K. Worden, Transmissibility of non-linear output frequency response functions with application in detection and location of damage in mdof structural systems, International Journal of Non-linear Mechanics 46 (6) (2011) 841-853.

[9] D. E. Adams, C. R. Farrar, Application of frequency domain arx features for linear and nonlinear structural damage identification, Smart Nondestructive Evaluation For Health Monitoring of Structural and Biological Systems 4702 (2002) 134-147.

[10] D. E. Adams, C. R. Farrar, Classifying linear and nonlinear structural damage using frequency domain arx models, Structural Health Monitoring - An International Journal 1 (2) (2002) 185-201.

[11] L. Bornn, C. R. Farrar, G. Park, K. Farinholt, Structural health monitoring with autoregressive support vector machines, Journal of Vibration and Acoustics-transactions of the Asme 131 (2) (2009) 021004.

[12] L. Bornn, C. R. Farrar, G. Park, Damage detection in initially nonlinear systems, International Journal of Engineering Science 48 (10) (2010) 909-920.

[13] H. W. Chen, Modeling and identification of parallel nonlinear-systems structural classification and parameter-estimation methods, Proceedings of the IEEE 83 (1) (1995) 39-66.

[14] A. Novak, L. Simon, F. Kadlec, P. Lotton, Nonlinear system identifica- 
tion using exponential swept-sine signal, Ieee Transactions On Instrumentation and Measurement 59 (8) (2010) 2220-2229.

[15] M. Rébillat, R. Hennequin, E. Corteel, B. F. G. Katz, Identification of cascade of hammerstein models for the description of nonlinearities in vibrating devices, Journal of Sound and Vibration 330 (5) (2011) 1018-1038.

[16] G. Palm, Representation and approximation of non-linear systems .2. discrete-time, Biological Cybernetics 34 (1) (1979) 49-52.

[17] R. Hajrya, N. Mechbal, Principal component analysis and perturbation theory based robust damage detection of multifunctional aircraft structure, Structural Health Monitoring - An International Journal 12 (3) (2013) 263-277.

[18] T. Jolliffe, I., Principal Component Analysis (second edition), Springer, 1986.

[19] H. Golub, G., F. Van Loan, C., Matrix Computation (first edition), 1983.

[20] C. Davis, W. Kahan, The rotation of eigenvectors by a perturbation. iii, SIAM Journal on Numerical Analysis 7 (1) (1970) 1-46.

[21] R. Hajrya, N. Mechbal, M. Verg, Active damage detection and localization applied to a composite structure using piezoceramic patches, in: IEEE Conference on Control and Fault Tolerant Systems, 2010. 
516 [22] J. S. Uribe, N. Mechbal, M. Rébillat, K. Bouamama, M. Pengov, Probabilistic decision trees using SVM for multi-class classification, in: 2nd

518 International Conference on Control and Fault-Tolerant Systems, 2013. 


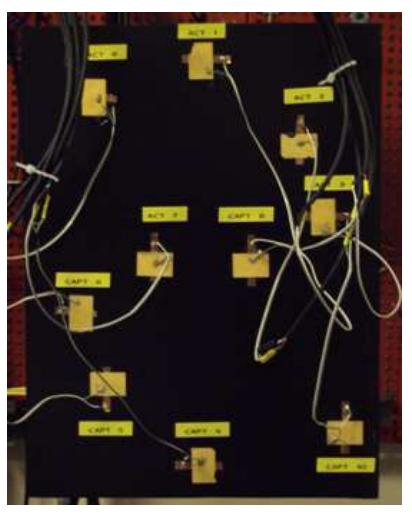

(a)

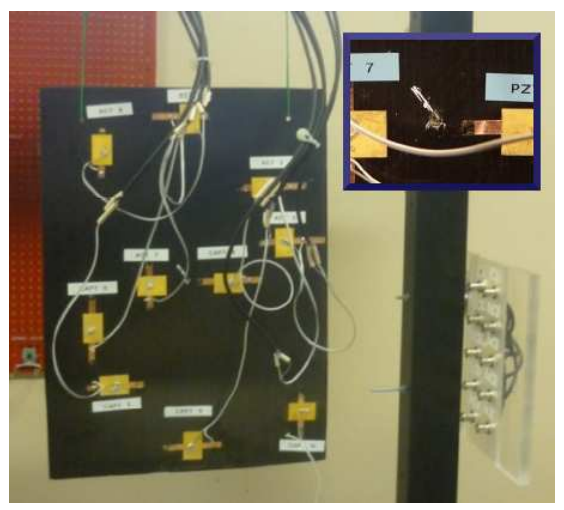

(b)

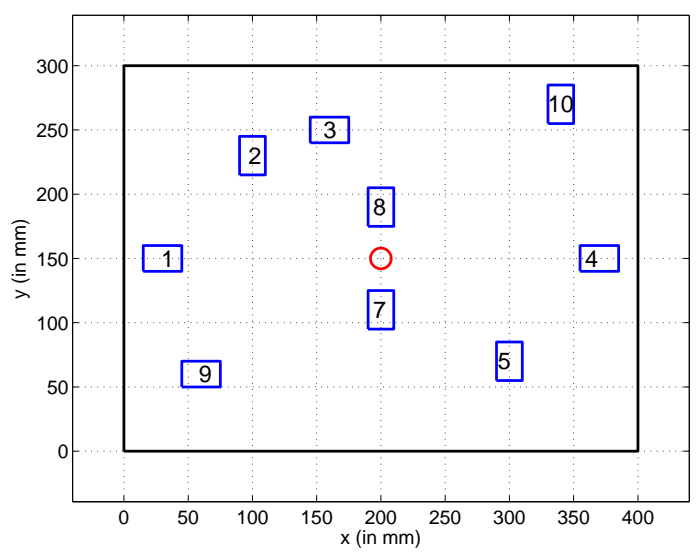

(c)

Figure 6: (a) Healthy and (b) damaged composite plates with a zoom on the impact damage. (c) Schematic representation of the plates under study (circle denotes damage position and rectangles stand for PZTs). 


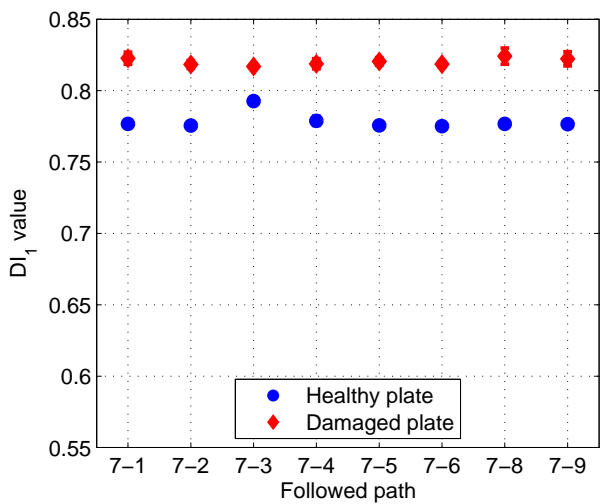

(a)

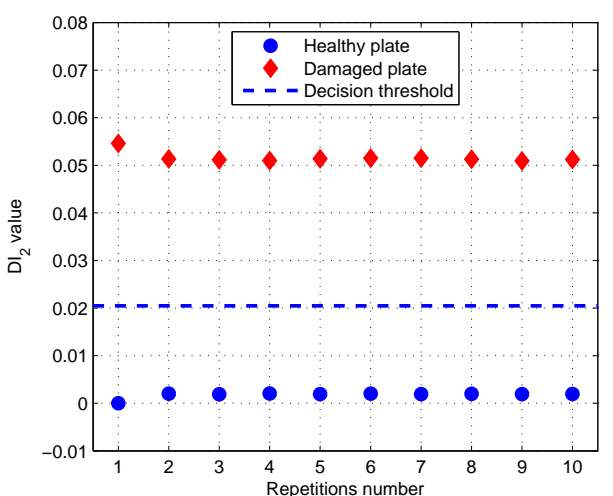

(b)

Figure 7: (a) Averages and standard deviations of $\mathrm{DI}_{1}$ values for the different paths starting from actuator 7 . (b) $\mathrm{DI}_{2}$ values for the different repetitions for actuator 7 and definition of the detection threshold.

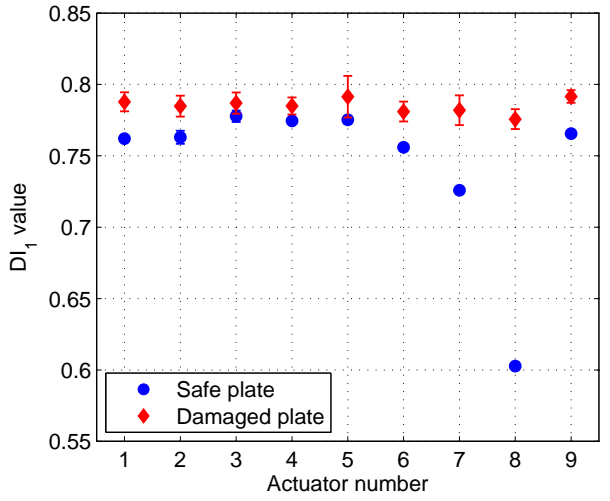

(a)

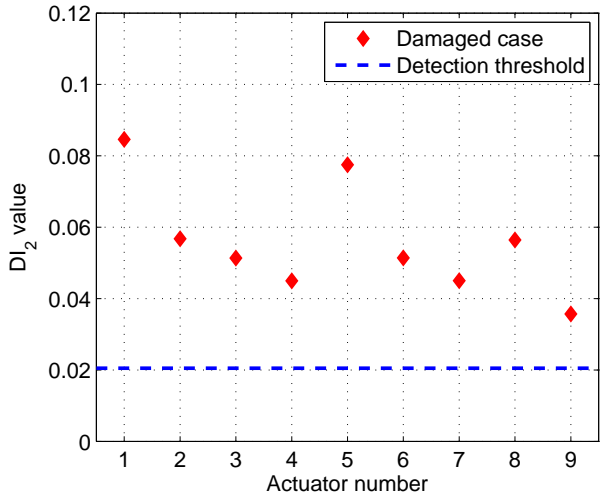

(b)

Figure 8: (a) Averages and standard deviations of $\mathrm{DI}_{1}$ values for the different actuators.

(b) $\mathrm{DI}_{2}$ values for the different actuators in comparison to the detection threshold. 